\title{
Masculinidades en la formación profesional: expectativas y sentidos del trabajo en un contexto de incertidumbre laboral
}

\author{
Masculinities in vocational education and training: expectations and the sense of work in a context of labor \\ uncertainty \\ Verónica Millenaar \\ Programa de Estudios sobre Juventud, Educación y Trabajo del Centro de Investigaciones Sociales del IDES/ \\ CONICET (PREJET-CIS-IDES/CONICET), Argentina \\ veronicamillenaar@gmail.com
}

\section{Resumen:}

\begin{abstract}
El artículo analiza diferentes expectativas de formación y sentidos del trabajo de varones egresados de Centros de Formación Profesional (FP). Se basa en una investigación doctoral, de abordaje cualitativo, en la cual se reconstruyeron trayectorias de jóvenes egresados/as de tres centros de FP situados en la Ciudad de Buenos Aires y conurbano norte y noroeste de la provincia de Buenos Aires, durante los años 2008 y 2013. El artículo muestra diferentes masculinidades, así como la construcción de múltiples sentidos de la FP entre los jóvenes con el horizonte puesto en complejizar el rol de dichas instituciones como dispositivos de socialización ocupacional y de género.
\end{abstract}

Palabras clave: Masculinidades, Formación Profesional, Jóvenes, Argentina.

\section{Abstract:}

The article analyses different expectations on training and senses of work shared by male graduates of Educational and Vocational Training Centers (VET). It is based on a doctoral research, with a qualitative approach, which analyses trajectories of young graduates of three VET centers located in the City of Buenos Aires and the north and northwest of the province of Buenos Aires, during the years 2008-2013. The article shows different masculinities as well as the construction of multiple senses of VET as a contribution to problematize the role of such institutions as mechanisms of occupational socialization and gender.

KEYWORDs: Masculinities, Educational and Vocational Training, Youth, Argentina.

\section{INTRODUCCIÓN}

La formación profesional (FP) en la Argentina es uno de los niveles educativos que se orienta a la transmisión de saberes socio-laborales, y que a lo largo de su historia ha tenido un papel relevante en la formación y cualificación de los y las trabajadores/as. Se ha planteado que, históricamente, su oferta ha reflejado, casi sin cuestionamientos, la división sexual del trabajo. Las discriminaciones del mercado laboral en relación al género se reflejan en el modo en que se organiza el sistema de FP, que se caracteriza por diferenciar su oferta formativa para varones y mujeres, presentar discriminaciones hacia las mujeres en cursos típicamente masculinos y producir inserciones laborales de diferente calidad en varones y mujeresuna vez finalizados los cursos-(Millenaar y Jacinto, 2015).1 Así,-del mismo modo en que ocurre en todo el conjunto de la educación técnico-profesional (ETP) - en el nivel de la FP subsisten sesgos sexistas que se reproducen acríticamente y que incluso permanecen con pocos cuestionamientos en el actual contexto en donde la agenda de género ha cobrado enorme relevancia.

Desde esta evidencia, una de las preguntas que apremia es aquella referida a las formas en que se producen y reproducen las desigualdades de género en dicho nivel, que podría contribuir a delinear estrategias para producir mayor equidad. Los aportes de la teoría de género, en este sentido, son extensos y permiten reconocer el modo en que los "códigos de género" (Subirats, 1986), presentes en las instituciones educativas, inciden en 
cómo, alumnos y alumnas, llegan a una identificación personal en términos de varón y mujer. Pero a pesar de que es posible reconocer una afianzada reproducción de códigos de género hegemónicos en la FP, también es necesario resaltar la heterogeneidad de experiencias biográficas y subjetivas de quienes llegan a esas aulas. Por ejemplo, en una investigación anterior (Millenaar, 2014) se mostraba que el alumnado que se acerca a los centros de FP aspira a encontrar experiencias y recursos que varían significativamente según sus edades, capital educativo, lugar de residencia, si cuentan o no con experiencia laboral, o si son madres/padres. Estas demandas suponen desafíos contrastantes para las instituciones, en el sentido de cómo intervenir desde una perspectiva de género. Se señalaba también, que, en el caso de las mujeres, no supone el mismo el trabajo institucional si ellas se acercan para adquirir una certificación y profesionalizarse, o si lo hacen para encontrar un espacio de sociabilidad y poner a circular la palabra, o para para aprender una nueva actividad y "testear" una vocación no del todo definida.

De acuerdo con esta evidencia, la lectura sobre el modo en que se reproducen las desigualdades de género en las instituciones de FP no puede ser nunca lineal, binaria y dicotómica, sino que exige agudizar el análisis para no perder de vista los múltiples "encuentros" que se entablan entre las subjetividades y las propuestas institucionales. En esta dirección, se busca mostrar, particularmente para el caso de los varones, las diferentes motivaciones respecto de la formación y sentidos del trabajo que ellos presentan, poniendo en evidencia la existencia de matices y contrastes en las identificaciones de género masculinas. Estos contrastes, claro está, desafían a las instituciones en sus estrategias educativas en pos de la equidad de género: mientras que en muchos centros persiste la idea de que a la FP llegan aprendices y obreros a formarse bajo los códigos masculinos hegemónicos, las experiencias reales de los alumnos varones distan bastante de esa construcción imaginaria. El hecho de elegir mostrar particularmente las masculinidades busca responder a cierta ausencia del análisis de las experiencias de los varones en los estudios de género sobre la ETP; estudios que ya de por sí son escasos en nuestro medio.

Así, el artículo presenta resultados parciales de una tesis doctoral (Millenaar, 2017a), que tuvo el objetivo de analizar trayectorias educativo-laborales de jóvenes para reconocer el papel que en ellas juegan las instancias educativas de formación para el trabajo, asumiendo la multiplicidad de factores que se conjugan en la configuración de dichos recorridos y experiencias. Metodológicamente, la investigación tuvo un abordaje cualitativo, con un trabajo de campo extenso en el cual se reconstruyeron trayectorias de jóvenes egresados/as de tres centros de FP ubicados en la Ciudad de Buenos Aires y el conurbano norte y noroeste de la provincia de Buenos Aires, durante los años 2008 y 2013. ${ }^{2}$ Este artículo se basa en 30 entrevistas a varones de entre 18 y 32 años de edad, ${ }^{3}$ con el objetivo, aquí, de analizar tanto las expectativas de formación como los sentidos del trabajo y las vinculaciones que se reconocen en sus discursos en relación a sus identificaciones como varones, jóvenes y trabajadores.

A continuación, el artículo aborda la discusión que aporta la literatura acerca de las masculinidades en la FP, para luego avanzar en el análisis de los jóvenes entrevistados. Se muestran, así, diferentes formas de ser varón y la construcción de múltiples sentidos de la FP entre los jóvenes con el horizonte puesto en complejizar el rol de dichas instituciones como dispositivos de socialización ocupacional y de género.

\section{Masculinidades en la FP: ¿Tiempo de cambios?}

En su trabajo emblemático, Paul Willis (1998 [1977]) estudió el modo en que jóvenes varones de sectores obreros, en sus interacciones escolares, erigían y consolidaban una cultura contra-escolar, basada en su identidad de clase obrera y de género masculino, que tendía a desmerecer el rol del saber intelectual y a valorizar el trabajo manual. Esta cultura contra-escolar favorecía la orientación de sus carreras profesionales hacia el empleo fabril, volviendo evidentes las imbricaciones entre los modos de configuración identitaria con procesos de reproducción cultural y social de mayor alcance. El autor mostraba, en la sociedad salarial industrial y de pleno empleo inglesa de mediados de los ' 70 , cómo los sectores populares obreros encontraban, 
en el espacio fabril, el ámbito donde afirmar la propia masculinidad. Estos eran trabajos en los que se valoraba la fuerza física y donde podía ejercerse, al mismo tiempo, una gestualidad de la resistencia, desde la producción de una subjetividad ruda y que asumía riesgos.

También, desde la literatura inglesa, la FP ha sido analizada en esta clave en relación a su rol como dispositivo de socialización ocupacional y de género. Los estudios de Inge Bates (1991) y Beverly Skeggs (1997) se enfocaron en las trasformaciones a nivel de las subjetividades de jóvenes mujeres, mostrando que, si la escuela cumplía un rol general en la reproducción de clase y de género, esto requería un ajuste mucho más preciso en el marco de la formación profesional, que se encontraba dirigida a determinada inserción ocupacional en concreto. Este ajuste no solo se vinculaba a la incorporación y apropiación de saberes, sino a un cambio actitudinal que, en un proceso que ellas describen como "dramático", garantizaba que las jóvenes se auto-limitaran en sus expectativas y aspiraciones vocacionales, para cumplir y aceptar — dadas su posición de clase y de género - los requerimientos reales de los empleos disponibles (feminizados, exigentes, de bajos salarios y malas condiciones laborales).

Trabajos recientes plantean nuevos aportes con relación a los aprendizajes, las modificaciones actitudinales y el reforzamiento de las identidades de género que se producen en el marco de los dispositivos de formación laboral. En el caso de los varones, las modificaciones actitudinales y el reforzamiento de las identidades de género experimentadas a lo largo de la instancia formativa se orientan hacia la consolidación de las normas de la virilidad. Esto es: la habituación de los cuerpos al esfuerzo físico, la tolerancia al dolor y el sufrimiento, el acostumbramiento al trato rudo entre compañeros y con los superiores, la disposición a desafiar el peligro, la negación del miedo, y el menosprecio a todo lo considerado femenino. Por su parte, se muestra que aquellos varones que eligen formarse en empleos que no son típicamente masculinos padecen la desestimación social por su incumplimiento con el rol de proveedores (Lamamra, 2011).

Las teorías sobre la masculinidad plantean que, a lo largo de la historia, se ha impuesto un ideal masculino vinculado a la potencia y la superioridad; en contraste a la debilidad y sumisión asociada a la posición femenina. La masculinidad se afirma en el ejercicio del dominio, el control, la dureza y la expresión de la insensibilidad afectiva. Asimismo, como consecuencia de la división sexual del trabajo, ese ideal demanda la provisión material y protección simbólica de las mujeres e hijos/as, que se realiza a través de trabajo extradoméstico. Por supuesto, el mismo se erige en el imaginario como una imagen de masculinidad hegemónica que orienta (aunque no determina) la afirmación de las relaciones de género de los varones en un contexto patriarcal. Dicho ideal orienta e interpela, de diferentes maneras, a los individuos concretos constituyéndolos como sujetos. No obstante, ello no supone la posibilidad efectiva de realizarse plenamente al modo de esa imagen imperante y, en rigor, la experiencia de identificación varía entre los varones, reconociéndose formas diversas de la masculinidad (Connell y Masserschmidt, 2005).

Esto se vincula con lo que Rita Segato (2003) postula cuando habla de la "gramática de género". Para ella, dicha gramática es el mapa cognitivocon el que los sujetos operan. La interpretación de ese mapa cognitivo es siempre una instancia individual y puede ser bastante aleatoria y accidentada: no es posible pensar las identidades de género como algo acabado, sino como un proceso en permanente transformación.

Cabe señalar que los contextos de crisis de empleo y mayor precarización laboral obligan a repensar el modo en que los varones, en un marco de mayores dificultades de acceso al empleo, se relacionan con esa masculinidad hegemónica. Al respecto, se han señalado las consecuencias psicológicas y sociales en los varones cuando se ven imposibilitados a cumplir el mandato hegemónico del ser varón (Jiménez Guzmán y Tena Guerrero, 2007).

En este artículo se centra el foco en jóvenes, entre quienes los problemas de empleo aparecen con más virulencia. Desde hace décadas, los estudios laborales ponen en evidencia peores indicadores de desempleo y precariedad laboral cuando se trata de la población joven. La desigualdad respecto de la población adulta incluso subsiste en períodos de reactivación económica, evidenciando las dificultades que atraviesan los grupos juveniles en sus procesos de inserción laboral (Pérez, 2016). 
Marina Medán (2013), en un estudio sobre masculinidades adolescentes,postula que, cuando las condiciones económicas y culturales no permiten cumplir con una masculinidad hegemónica asociada a la figura del varón proveedor tradicional mediante un trabajo estable, la forma de resolver esa contradicción es — tal como señala Robert Connell (1995) - abrazar a la marginalidad y al estigma y de esta manera volverlos redituables, al inspirar miedo y temor. Frente a la posibilidad de ser considerado "cobarde", los varones recurren a la fuerza física (a las "piñas"), como un capital valorado, frente a la ausencia de otros capitales económicos y educativos (Medan, 2009, 2013). Esta valorización del "capital físico" se vincula a los requerimientos laborales de empleos precarios, inestables y desprotegidos que, en sus exigencias físicas, demandan el poder "bancarsela". ${ }^{4}$ Este aspecto es interesante para abordar los procesos de vinculación con el empleo entre jóvenes varones de sectores populares, entre quienes tiene relevancia el hecho de mostrarse en el mundo laboral como trabajadores resistentes, fuertes, que desmienten el dolor, frente a empleos que exigen fuerza física y sacrificios, a veces extremos (Silba, 2011). Así, el ideal del capital físico se vuelve entonces un elemento central, que ha sabido utilizarse en la gestión de la fuerza laboral, en ciertos territorios de la informalidad típicamente masculinos del mercado de trabajo.

Asimismo, la sociabilidad de la calle y el barrio (en contraste con el espacio doméstico) es un elemento de identificación identitaria relevante, así como un factor de segregación de género (Bourgois, 2010). En el caso de los varones, la calle constituye el espacio físico y simbólico donde autoafirmar la masculinidad, sobre todo cuando el empleo estable, que permitiría realizar el ideal de proveedor, es de difícil acceso.

En este marco, los centros de FP, ofrecen a sus alumnos y alumnas saberes vinculados a los requerimientos del mercado de trabajo. Históricamente, la FP se encargó de formar trabajadores masculinos a través de códigos de género vinculados al ideal de la superioridad, la fuerza, la provisión, la protección. No obstante, en el actual contexto de precariedad laboral, los jóvenes varones se acercan a los cursos con intereses diversos, compartiendo representaciones de sí como varones, trabajadores y jóvenes que, en ocasiones, distan de aquel ideal patriarcal; pero respecto del cual, inevitablemente, deben entablar algún tipo de relación (resistiéndolo, impugnándolo o adhiriendo a él), porque está lejos de haberse "caído".

A partir del análisis de sus trayectorias educativo-laborales, se presentan, a continuación, tres grupos de varones que se diferencian en relación a sus discursos en torno a sus expectativas de formación, sentidos del trabajo e imágenes de sí mismos como varones y trabajadores. En un primer grupo, el trabajo es la esfera que adquiere centralidad en sus trayectorias y, por lo tanto, en sus discursos, es la profesionalización en el oficio el proyecto que se prioriza. En un segundo grupo, las redes de sociabilidad barrial adquieren relevancia en los sentidos dados a la formación, y se vinculan a la representación que tienen de sí mismos como jóvenes varones y trabajadores. En un tercer grupo, el estudio adquiere centralidad, y por ello, las expectativas de formación y los sentidos del trabajo se vinculan al esfuerzo más general de continuar estudios superiores. Cabe señalar que todos los jóvenes analizados son egresados de cursos de FP en diferentes especialidades (carpintería, soldadura, ventas, gastronomía, mecánica de motos), en centros que, en su mayoría, reciben población de sectores populares.

\section{3. "YA NO PODÉS HACERTE EL GIL": VARONES CON EL PROYECTO CENTRADO EN EL OFICIO}

De los varones entrevistados, un primer grupo, ${ }^{5}$ puede presentarse como el más cercano al ideal masculino hegemónico: sus identificaciones de género no cuestionan ni critican la división sexual del trabajo. Si bien ninguno se autopercibe como machista o conservador - y en la situación de entrevista prefieren argumentar desde un lugar de enunciación "políticamente correcto"-, la indagación en profundidad respecto de estos temas muestra una percepción de sí mismos más cerca del mandato tradicional. Es decir, se perciben como varones que, en la familia actual, o en el futuro, cuando la conformen, tendrán la responsabilidad por la provisión. De hecho, su aspiración a profesionalizarse y crecer en la carrera laboral entra en sintonía con este mandato que aspiran a cumplir. El empleo extra-doméstico es su prioridad y su eje de organización vital. 
Incluso, se asume que los cambios sociales que afectaron este esquema tradicional de la división sexual del trabajo ocasionaron problemas en las familias y su vinculación. Así lo expresa uno de los varones del grupo:

Estaría bueno que sea así, ¿no? Que la mujer pueda criar a sus hijos en la casa, y el varón sea el que traiga la comida todos los días. Pero en la sociedad que estamos viviendo no se da. Entonces, ahí está la raíz de todo. La madre trabaja, el padre trabaja y cuando vuelven... al hijo lo cría un tercero (...) Y yo pienso que en realidad lo ideal sería uno tener un buen sustento, y que puedas mantener a la mujer, y que la mujer se ocupe de la casa (Alejandro, 25 años).

Por supuesto, no todos los varones de este grupo manifiestan estas ideas tan explícitamente, pero en su mayoría dejan entrever similitudes con esta visión. En otros casos, el discurso se centra más en la idea de que ellos son protectores y quieren cuidar de sus mujeres.

Yo soy un tipo protector con mi pareja. Si fuera por mí, y si ella lo desea, que estudie toda la vida y yo siempre le daría una mano con lo que sea, para que siga estudiando, creciendo, si no quiere laburar que no labure. Por ahí eso es un pensamiento machista, me entendés, o no. Es una mezcla (David, 22 años).

En ese par "provisión/protección" se reconoce la idea de que el mercado de empleo está dividido naturalmente. Existen empleos que están dirigidos a mujeres, y otros a varones. Esta división se encuentra asociada a las costumbres, pero también a atribuciones físicas, como la fuerza. Así, la división sexual del trabajo se refleja, por una "cuestión natural" en el mercado de empleo, en el cual las mujeres realizan determinadas actividades y no otras.

- ¿Por qué pensás que están divididos los trabajos de hombres y mujeres?

- Es una cuestión natural (...) a las mujeres les gustan las cosas que les gustan a ellas, no encajan en el ambiente de hombres (Alan, 18 años).

Incluso, en algunos se manifiesta la percepción de que un curso de FP típicamente masculino, como en el caso de la mecánica, es la oportunidad para sentirse "más varón". Entre otras cosas, porque permite incorporar un lenguaje que entre pares es conveniente saber manejar, para no ser considerado un extraño o un diferente. David cursa mecánica precisamente para no ser considerado un "bicho raro" y estar mejor plantado en lo que él considera "la utilidad del hombre".

-Yo me sentía un bicho raro en las típicas charlas de hombres... viste que tienen que ver con la mecánica, porque yo era como un inútil. Pero... ahora pienso que puedo cortar, que puedo poner partes, que para mi criterio puede servir... y eso está bueno. -Estás mejor plantado en este rubro de la mecánica...

-Sí, obvio. O mejor plantado en lo que es la utilidad del hombre, particularmente, porque es importante (David, 22 años).

Los jóvenes que pertenecen a este grupo se caracterizan por mostrarse fuertemente comprometidos con el trabajo. En efecto, el compromiso con el trabajo resulta un elemento significativo en la toma de decisiones y en los pasos dados a lo largo de sus historias laborales; y resulta un rasgo que los constituye en términos de su identidad.

Yo empecé a trabajar de muy chico, para colaborar con mi familia, y nunca paré... para mí el trabajo es todo. Yo lo veo como un bienestar, o sea si uno no trabaja no puede tener nada, no puede proyectar nada... o para valerte por vos mismo... no sé... no sos nadie si no trabajás (Lautaro, 20 años).

Para ellos, el trabajo es una tarea de vital importancia asociada al bienestar económico, psíquico y social. El esfuerzo y los sacrificios que se realizan en pos de sostener el trabajo son frecuentemente señalados. Si bien no todos cuentan con el trabajo ideal, sin embargo, se encargan de dejar en claro que en el trabajo son responsables, cumplidores, serviciales y emprendedores. El temple con el que enfrentan la actividad diaria encuentra su sostén en ese plan de carrera profesional imaginado y en la representación compartida de que el trabajo "es todo".

Siempre busco avanzar en la vida, en cuanto a lo profesional o como persona (...) Y de la importancia del trabajo en la vida de uno ¿no? De no tomarlo como una obligación, sino de que sí, que es necesario, es necesario trabajar, pero también saber 
cómo llevarlo cotidianamente, porque siempre tenemos distintas situaciones en el trabajo, las que nos gustan, las que no nos gustan, como todo, pero sabés cómo llevar esa situación (Francisco, 25 años).

En cuanto a sus expectativas de formación, aparece en este grupo el deseo de formarse en un oficio que eligen para ellos y que les gusta. En algunos casos, se trata del oficio que ya vienen desarrollando; es decir, la oportunidad laboral les generó el impulso a buscar formarse en el oficio. En muchos otros, se trata de un interés que aparece en un determinado momento y que los "captura": reconocen en ese oficio un camino de construcción a largo plazo que los interpela. Cuando saben que "ese" es el oficio, ya no "pueden hacerse los giles". A partir de ese momento, se trata de responsabilizarse como trabajadores, proveedores y varones. Entonces, la identificación con el oficio es la vía por la cual terminan sintiéndose cómodos en su adhesión al ideal masculino hegemónico. La frase "No podés hacerte el gil" representa ese movimiento subjetivo: el oficio trae consigo la vía para realizarse como varones trabajadores. Pero eso, también, involucra el hecho de hacerse cargo y responsabilizarse ante esa evidencia.

-Lo que pasa es que ya es algo de muy de chico, y llega un momento en el que te tenés que poner las pilas y decir "Bueno vamos a hacer algo". Ya está. A los 25 años tenés que recibirte de algo, hacer algo. Y bueno, si te va mal, y bueh... por lo menos, lo intentaste.

-¿Vos te sentís mecánico, Alejandro?

-Sí, sí, me gusta (Alejandro, 25 años).

El curso me lo puse a hacer porque esto me fascina. Con este curso siento algo muy particular: que no me es un problema sentarme a estudiar. Y la verdad es que no lo sentí con las demás carreras. Me parece que me tengo que dar la oportunidad acá. Me influye mucho más lo técnico. Y cuando tenés la oportunidad de verlo, no podés hacerte el gil (David, 22 años).

De algún modo, lo que ocurre en este grupo es que el oficio específico comienza a marcarles el rumbo laboral; y en ese rumbo, afirman su identidad como trabajadores varones. La profesionalización que adquieren en el curso de formación con el que se vinculan -aunque no solamente sucede únicamente a partir del cursoresulta un anclaje subjetivo a un recorrido laboral específico.

-Vos no podes ir a buscar otro trabajo y decir "Che, dame cualquiera”, porque por ahí te dan uno que es para cualquier otra cosa. Uno ya está enfocado en algo. Yo quiero, por decírtelo así, morirme en soldadura, porque a mí me gusta el oficio (Sebastián, 19 años).

-Siempre me gustó lo que es mecánica.

-¿Cuándo empezaste a tener el taller de motos en tu casa?

-A mitad del año pasado. Había terminado el curso y tengo varios amigos que tienen moto, y así de boca en boca... fui arreglando motos y sigo arreglando acá a los vecinos del barrio. Y ahora lo que tengo pensado yo es ponerme una casa de repuestos, aparte el taller... Y tengo pensado estudiar en una escala más grande... Porque lo más importante para mí es esto... lo que tengo pensado... las motos. Es mi proyecto (Pablo, 19 años).

Cabe señalar que, luego de los cursos, además del reforzamiento del compromiso con un proyecto de trabajo, la mayor parte de los entrevistados evidencian efectivamente un cambio en sus trayectorias laborales luego del egreso. En aquellos que logran insertarse en el mercado laboral formal, dicha inserción constituye una oportunidad de mucho valor en relación a sus trayectorias laborales, sobre todo considerando las experiencias de trabajo anteriores. Los jóvenes con esta nueva situación perciben haber cumplido (y traspasado) sus expectativas iniciales.

De este modo, en este grupo, la expectativa de profesionalizarse en el oficio se vincula al discurso tradicional compartido en torno al rol del varón como trabajador proveedor y protector de sus familias. Por lo tanto, el sentido dado a los cursos, además de ser la vía para responsabilizarse en relación con su identidad laboral, también los afirma en términos de su masculinidad. 


\section{4. "UNOS TRABAJAN DE TRUENO, y ES PARA OTROS LA LLOVIDA": VARONES CON EL PROYECTO CENTRADO EN LA SOCIABILIDAD}

En un segundo grupo de varones, las identificaciones de género también son tradicionales, pero con diferencias respecto del primer grupo. Estos jóvenes, ${ }^{6}$ sobre todo en la construcción de una imagen de sí mismos a futuro, también como en el primer grupo, se piensan como proveedores del hogar. En ese sentido, si bien comparten un discurso en torno a la importancia de la igualdad en las tareas, en su rol de padres de familia se imaginan cumpliendo el clásico destino masculino. No obstante, el papel de proveedores a futuro se percibe como un anhelo difícil de lograr en relación a las condiciones laborales del presente, al mismo tiempo que se resiste por el padecimiento físico que involucra.

En este grupo, se comparte la idea de que el trabajo es una actividad obligada, vinculada a la necesidad de subsistencia material. El trabajo está asociado a una actividad no placentera y contrasta con otras actividades que sí se disfrutan (la música, el gimnasio, "los pibes"). El displacer se encuentra asociado a las condiciones del mercado de trabajo, que los jóvenes del grupo, caracterizan como de explotación. Si pudieran hacerlo, preferirían no trabajar o vincularse de otro modo con el trabajo y el placer.

Y porque el laburo es sacrificado, horas y horas, para ganarte el mango... El laburo es un sacrificio... Pero yo le escapo al trabajo que hace que no vivas y te rompas el lomo..., prefiero buscar algo que a mí me guste, que disfrute (Samuel, 18 años).

No es casual que Mariano, por ejemplo, se identifique con las "Coplas del Payador Perseguido" de Atahualpa Yupanqui que afirma en su letra que "está perdida la vida, trabajando en campo ajeno" 7

\footnotetext{
El otro día estaba escuchando un tema de Jorge Cafrune, "Payada de un gaucho perseguido" o algo así y el tipo decía "a mí no me gusta trabajar en campo ajeno". Tiene razón. Muchos que trabajan de sueldo, pero la guita les cae a otros... Yo no voy a dejar mi vida laburando 12 horas en un lugar para que me paguen 5000, 6000 pesos, volver amargado a mi casa, en vez de volver contento, estar con mi mujer, comer, ver una película y qué sé yo, llegar a mi casa con cara de ojete, reventado de cansancio, y hacer algo que no me gusta, para que el grosso de la guita se la lleve el dueño (Mariano, 32 años).
}

En este grupo se percibe el padecimiento que deriva de aceptar los empleos circundantes orientados a varones de clases populares. Por un lado, se reconoce la carga de virilidad que portan (son trabajos rudos, en la intemperie y exigen de la fuerza física), pero por otro, se reconoce en ellos sufrimiento y desgaste. Un ejemplo ilustrativo de la ambivalencia que generan estos empleos (que por un lado implican aceptar el mandato de "bancarselos" y, por el otro, cuestionarlos), se reconoce en el relato de Lucas. Este chico, proveniente de una familia de albañiles, comenzó a trabajar muy jovencito. A los 15 años, un vecino del barrio abrió un local de venta de accesorios para mascotas y le pidió a Lucas que trabajara con él. En el marco de ese trabajo, Lucas realizó un curso capacitación en "Baño y peluquería canina", gracias al cual hoy tiene un carnet de especialista en esa actividad, que muestra con orgullo. Relata que el trabajo le gustaba. Sin embargo, a los 18, cumpliendo con cierta tradición que existe en su familia, dejó ese trabajo y entró en una obra como albañil, por más dinero. Si bien desde niño había querido ser albañil "como su papá y su abuelo" y consideraba que ese trabajo le permitiría afirmar mejor su masculinidad que ejerciendo como peluquero canino, Lucas, no obstante, hoy está arrepentido, sobre todo por el esfuerzo físico que implica ese trabajo.

Estaba por el tercer piso la semana pasada, iba caminando así, porque estábamos en la obra y a la par, al lado, hay dos oficinas y me pongo a mirar... y miraba cómo pasaban todos con camisa, tomando mate, se cagaban de risa, y yo decía "y yo acá todo sucio, húmedo, haciéndome mierda la espalda, levantándome a la mañana”. (...) Pero para mí el trabajo del hombre era el de la obra. Y ahora no quiero más esto” (Lucas, 18 años).

Con trabajos inestables e imposibilidad de acumulación a través de empleo fijo, los varones entienden que el cumplimiento del papel de proveedores se encuentra obstaculizado. Así, el mismo se percibe como un ideal, para cumplir en el futuro. Mientras tanto, en el presente, la afirmación de la masculinidad se garantiza a través de la propia aceptación en el grupo de pares o en el espacio de la calle, en donde los varones se miden fuerzas. 
Como han planteado diversos autores, el riesgo, el "tener aguante", son atributos generalmente asociados al mundo masculino (Alabarces y Garriga Zucal, 2008), que los jóvenes de este grupo vinculan con la "calle" entidad no solo física sino también de enorme carga simbólica. La "calle” no es solo un espacio de aprendizaje, que permite hacerse adulto ("y ponerse pillo" ${ }^{8}$ ), sino que también, tal como señaló Bourgois (2010) el hecho de saber habitarla ("de saber caminarla") es la oportunidad de hacerse respetar por el grupo de pares. Las redes de sociabilidad que ofrece la calle permiten a los varones de este grupo la afirmación de su masculinidad, principalmente en relación a la gestualidad viril: ser fuerte, rudo, saber arriesgarse, saber pelear y defenderse.

“-Y si, acá me rescaté... Porque en la calle... (...) No me gustaría hablar mucho... pero si querés te digo, si querés te digo la verdad... [y lo que sigue, lo dice rapeando]:

En la esquina me conocen, yo vivo en la calle,

soy de la Rocha, soy Lucas Mansilla,

todos los fines de semana, viernes, sábado, domingo

vamos a la villa, vamos a comprar una pastilla.

Ando re empastado, vamos a comprar,

tengo bondi con éste, con el otro, tengo bondi con el de allá.

-(Silencio) Fuerte...

-El sábado este que pasó yo fui a la fiesta de la virgen y a un amigo le hicieron así con un cuchillo. Al otro día, agarraron todos los fierros y se fueron al fondo a tirar tiros (...) La calle enseña, te ponés pillo (...) pero yo me hago respetar" (Lucas, 18 años).

No obstante, los jóvenes también comprenden que la calle no es un espacio socialmente legitimado como una institución "de inclusión". En su imaginario, la inclusión legítima se reconoce en las actividades tradicionales, bien conceptuadas, del trabajo y la educación. De este modo, el curso de formación significa para ellos la oportunidad de acercarse a una institución de mejor concepto social que la calle.

"Ponerse las pilas" y "rescatarse" 10 son expresiones comunes entre los jóvenes de este grupo porque se comparte la idea de que tanto el trabajo, el estudio, como la responsabilidad adulta de la provisión, son todas esferas incompatibles y en tensión con la experiencia de sociabilidad de la calle. Los cursos de FP suponen entonces, un modo alternativo de sociabilidad, más legitimado socialmente. Este es el caso, por ejemplo, de Rodrigo y de Bruno, que, de acuerdo a sus relatos, la participación en los cursos supuso una transformación en sus vidas, que hasta ese momento estaban vinculadas al consumo problemático de drogas y al delito. Así, en el caso de Bruno, esta situación ya le había hecho perder dos empleos: en ambos, fue despedido acusado de estar vinculado a la droga. Bruno se encontraba sin una afiliación educativa ni laboral antes de decidirse a participar de la FP. La participación en el curso le permitió adquirir nuevas experiencias y compensar su desafiliación a instituciones tradicionales. Para Rodrigo, la participación en el curso le permitió dar un vuelco y cambiar ("ponerse las pilas" en su expresión). De acuerdo a su visión, el centro de formación es una oportunidad para la juventud, pero también para el barrio en su conjunto, porque se constituye en un punto de referencia para aquel que necesite contención y ayuda:

Y ahí empecé a cambiar, cambié un montón, gracias a esto, al curso. A mí me ayudó muchísimo, cambié un montón y cambió el barrio gracias a este centro (...) Sí, para mí fue justo dar en el clavo. Porque vos ves los chicos que vienen al curso, la forma en que hablan y te vas a dar cuenta que el curso fue importante para todos. Vos, más que nada que hacés los reportajes, te vas a dar cuenta la importancia de esto (Rodrigo, 26 años).

La intención de "ponerse las pilas" a veces no sucede inmediatamente luego del curso, sino que es una expresión de deseo para más adelante. En este sentido, los jóvenes de este grupo comprenden que su presente laboral desagradable podría revertirse en el caso de que terminaran el secundario, siguieran haciendo cursos y se esforzaran. En este grupo los obstáculos respecto de sí mismos generalmente se ubican externamente: en las malas condiciones de trabajo, la mala calidad educativa, la falta de oportunidades, etc. Sin embargo, el "rescate" que permite el curso ocurre cuando los jóvenes se vinculan con la institución de formación, y 
obtienen recursos y nuevos saberes que les permiten percibir un mayor "control" respecto de su biografía. Hacer un curso de FP permite en estos jóvenes adquirir saberes (y darse "maña”, ${ }^{11}$ como plantea Assusa, 2016) para enfrentar las condiciones de incertidumbre laboral. "Ponerse las pilas" es entonces ganar más autonomía en las propias decisiones y apostar a las consecuencias que de ellas deriven.

Lo que aprendí es a demostrar a que puedo llegar a dar más. Ya voy a ponerme las pilas, voy a terminar el secundario de una vez por todas, para ver si puedo... el año que viene voy a dedicarle al colegio, si lo hago, me anoto... por ahí sí, buscarme un laburo como la gente.... porque ya me cansé también, de tener laburos en negro que no te dan expectativa de nada, seguir laburando lo mismo y vos no tenés una obra social, no tener nada. Cobrar todo en negro no me gusta (Martín, 21 años).

Ahora bien, respecto de la formación, son específicas las visiones que surgen de los jóvenes, en relación a la oferta diferenciada de la FP de acuerdo al género (cursos orientados a varones y cursos orientados a mujeres, característica propia de este nivel educativo). Los chicos de este grupo defienden esa separación y encuentran en el argumento de la distracción el fundamento para justificarla.

-Y, cambia el trato, si tenés chicas en frente o no. Entre los muchachos nos hablamos de otro modo. Uno puede hablar si fue o no al puterío, o que estuvo con una minita. Con las chicas el trato es otro. (...) Y el tema del trabajo de las mujeres pasa por el hombre. Porque si una mujer quiere trabajar, el hombre tiene que comerse su orgullo. Tiene que dejarla. Es bueno que los dos trabajen.

-¿Conocés gente que no se coma el orgullo?

-Y sí, conozco gente más orgullosa que prefiere que la esposa esté en su casa. (...) Pero, ojo, también conozco mucha mujer que quiere salir a trabajar y en realidad es una manera de hacer trampa. Hay mucha mujer tramposa, también (Samuel, 18 años).

Como puede verse en la última cita, Samuel, a pesar de que coincide con la separación de los cursos, también deja en claro su apertura respecto de la igualdad de género. Sin embargo, esa apertura es para él, y sobre todo para su entorno, un ejercicio difícil, pues implica poner a un lado el "orgullo masculino". Este orgullo está derivado de la potestad que otorga ser el único proveedor del hogar. Si la mujer trabaja, el varón tiene que "comerse su orgullo" y además convivir con la incertidumbre de que la mujer pueda hacerle "trampa". ${ }^{12}$

De este modo, puede observarse que, en este segundo grupo, el mandato de género masculino vinculado al rol de proveedor sigue operando como un ideal en el imaginario. Sin embargo, las oportunidades reales de empleo impiden llevarlo a la práctica, al mismo tiempo en que, a diferencia del primer grupo, entre estos jóvenes se manifiestan mayores resistencias a aceptar trabajos en los cuales se padece desgaste físico e insalubridad. Así es que la afirmación de la masculinidad se produce a partir de las redes de sociabilidad barrial, que en ocasiones llevan a los jóvenes a involucrarse en prácticas desprestigiadas socialmente. Cuando se acercan a los centros de formación, los jóvenes de este grupo buscan una re-vinculación con instituciones socialmente legitimadas.

\section{5. "El discurso de las abuelas no va más": VARONES CON EL PROYECTO CENTRADo EN EL ESTUDIO}

En el último grupo de varones, ${ }^{13}$ el sentido central de sus acciones y elecciones se reconoce en el estudio y, principalmente, en la posibilidad de continuar con estudios superiores. A diferencia de los anteriores grupos, en éste, los varones en su mayoría completaron los estudios secundarios. Por el hecho de contar con mayor capital educativo, se perciben un tanto diferentes a los hombres de su entorno. Su deseo de estudiar, formarse, y su decisión de no darle prioridad al trabajo, los vuelve (de acuerdo a su propia percepción) "diferentes". De algún modo, el hecho de otorgar prioridad a una esfera que involucra un esfuerzo intelectual genera distancias con la valorización masculina del trabajo, sobre todo si éste involucra una actividad manual. Esta diferenciación de género (lo intelectual para las chicas, lo manual para los chicos) se observa incluso en las estadísticas educativas, que muestran una mayor presencia de mujeres en el nivel superior (Miranda y Corica, 
2015). Así, los varones que deciden otorgar prioridad al estudio por sobre el trabajo y buscar ampliar su horizonte respecto del papel de género que circula en su entorno, deben hacerlo manifestándose diferentes respecto de sus pares.

Un ejemplo revelador de la extrañeza respecto de sus pares lo manifiestan los jóvenes que estudiaron gastronomía, y debían asistir uniformados al curso (camisa blanca y pantalón negro), como un requerimiento para el aprendizaje. Ir de traje y "camisita” es algo disruptivo y extraño en los círculos masculinos en donde se mueven los jóvenes. No obstante, los varones portan la diferencia respecto de su entorno con cierto orgullo, como lo menciona Marcos:

- Yo entraba igual de uniforme no me importaba nada, la camisa y los zapatos. Pero sí, te miran, así, y te sentís diferente. O sea, en mi barrio no está tan impuesto eso de hacer cursos, estudiar en la facultad, es un barrio bastante cerrado, cuadrado digamos (...) Pasa eso: que te miran, y en la sociedad, lo diferente es raro. (...) Capaz yo lo re cuento con orgullo, a mí no me importa (Marcos, 19 años).

Incluso, esa percepción de extrañamiento respecto del entorno masculino es también reconocible en el ámbito laboral. Si en el primer grupo los varones buscaban en el curso una práctica confirmatoria de su masculinidad, los chicos de éste último grupo relatan vivenciar todo lo contrario: el hecho de ser aceptados por el entorno en sus puestos de trabajo es para ellos el ejercicio de "impostar" un código masculino que no comparten del todo. Así lo relata de un modo explícito Rodolfo, que trabaja como empleado de logística en una empresa, rodeado de compañeros varones:

-La verdad que nosotros somos todos varones, no hay ni una mujer. (...) Pero sí, de lo que se habla es de fútbol, de cosas así. Y cuando estamos en la carga del camión, es como un clásico el gritarle a una chica que pasa. Me doy cuenta que yo mismo cambio un poco en el trabajo. Es como que cambiás un poco la forma de hablar, para ser uno más. (...) Pero el ámbito es machista para mi gusto. Y eso lo veo sobre todo en los viejos, en los "gordos" como les decimos. Los más jóvenes no somos tan machistas.

-¿En qué sentido?

-Y no sé, por ejemplo... yo creo que los gordos no podrían aceptar una compañera mujer. Y yo no tendría ningún problema (Rodolfo, 20 años).

Así, en este grupo, se observa un discurso cuestionador respecto a los mandatos de género, como así también en relación a la división sexual del trabajo. Consideran que esas formas de pensar, como así también esas prácticas, quedaron en el "tiempo de las abuelas", y ese discurso, "no va más".

Yo soy tu novio, no soy tu dueño. Así que, andá y trabajá. Siempre fui así yo. Traté siempre de no ser tan machista. A mí papá lo escucho que le sale alguna que otra cosa machista y le digo "Eso suena muy feo. ¿Por qué tenés que hablar así?” (...) Es del tiempo de las abuelas (Gabriel, 20 años).

El hecho de necesitar trabajar para solventarse los estudios es un aspecto presente en algunos de los chicos de este grupo. Esto ocurre solo cuando se cuenta con algún interés o vocación ya definida. Entonces, el sentido de la formación es más instrumental y estratégico: se trata de buscar una inserción laboral suficientemente acorde con el proyecto principal de estudio o vocacional.

Uno de los jóvenes, Lucas B., muestra un interés muy marcado en continuar sus estudios superiores. La vinculación con la FP tiene el interés de intentar una inserción laboral que le permita "pagarse los libros" del profesorado. Máximo, otro de los jóvenes, mientras cursaba soldadura para obtener una salida laboral, se forma como músico y bailarín de folclore. Un caso similar es el de Rodolfo, con el dibujo. Vocación que le viene de niño y que hoy desarrolla como actividad placentera, mientras trabaja en un empleo formal en el rubro de logística.

De este modo, en este grupo, las expectativas de formación, están vinculadas a garantizar un proyecto de mayor alcance, vinculado a una vocación o recorrido educativo ya definido. En este marco, eltrabajo no es percibido por ellos como una actividad central en la propia vida. Se comparte la percepción de que el trabajo es una tarea necesaria, que debe ser realizada como posibilidad para concretar otros proyectos de importancia. 
De todos modos, cuando se define lo que se piensa y siente respecto del trabajo, se menciona el hecho de que permite la obtención monetaria. Trabajo (para la remuneración) y desarrollo personal (para realizarse en la vocación y estudio) se piensan como actividades separadas.

El trabajo para mí es una actividad para ganar plata. Ganar plata para algo en lo que te podés desarrollar. Pero por eso el trabajo no es necesariamente la actividad en la cual te pagan. Es como que el trabajo pasa por esas dos variables distintas, que pueden estar juntas o separadas. La remuneración, el desarrollo personal, digo (Rodolfo, 20 años).

Así, se observa que, en este último grupo, en donde se otorga centralidad a los estudios, la formación se percibe más instrumentalmente y en un sentido de acumulación de acreditaciones y aprendizajes para solventarse mejor el recorrido educativo que se anhela. Los mandatos de género son cuestionados y resistidos; aspecto que se vuelve por momentos un obstáculo para legitimar la propia virilidad en los grupos de pares menos afines a sus inquietudes.

\section{REFLEXIONES FINALES}

El género resulta una perspectiva de análisis fundamental para comprender las relaciones entre juventud, educación y trabajo, en un doble nivel analítico: el de las dinámicas institucionales, y su inserción en construcciones culturales y normativas más estables, fijas e invariantes, y el de las mismas prácticas biográficas (Scott, 2008). La categoría de género permite reconocer mandatos, estereotipos e idiosincrasias vinculadas a la división sexual del trabajo, a partir de las cuales se otorgan significaciones a la diferencia sexual.

En este sentido, el análisis de la desigualdad estructural de género debe complementarse con el registro de los códigos presentes en las instituciones, y con el modo en que varones y mujeres, en el marco de sus trayectorias educativas, se apropian, cuestionan o resisten dichos códigos, encarnándolos de modos diversos en sus propios recorridos. Los ideales hegemónicos de la masculinidad y la feminidad, no explican en sí mismos el modo en que se producen los recorridos juveniles entre la educación y el trabajo: en ellos, varones y mujeres toman decisiones, despliegan deseos, construyen sus vocaciones y elaboran una imagen de sí como varones y mujeres trabajadores/as de modos diversos.

A partir del análisis realizado, se pudo reconocer a jóvenes que adhieren y aceptan el ideal de masculinidad hegemónica, principalmente en relación al cumplimiento del rol de proveedor del hogar y "cuidador" de su mujer y su familia. Entre estos varones, la centralidad otorgada al trabajo se vincula directamente al cumplimiento de este papel, que les permite obtener recursos materiales pero también simbólicos. El trabajo resulta así el espacio de autoafirmación de la propia masculinidad. Los varones que comparten estos discursos entienden que el trabajo es una obligación, y asumen, responsabilizándose, su rol como trabajadores, a veces manifestando explícitamente el costo que ello involucra. Entre estos jóvenes, se percibe adhesión y poco extrañamiento y cuestionamientos a la segmentación de género. Los varones entienden que las diferencias con las mujeres son "naturales" y que incluso ellos son los responsables de que sus parejas o futuras esposas puedan "elegir" trabajar o no. Cumplir correctamente con el papel de género significa facilitarles a las mujeres su permanencia en el espacio doméstico desde un sentido de protección y provisión de cuidados.

En otro grupo de varones el papel de género tradicional también se manifiesta y comparte, pero circula discursivamente en términos de un "ideal" a alcanzar, que no se puede ni se quiere cumplir en el presente. Se trata de aquellos varones vinculados con mayores interrupciones y menores "lealtades" al trabajo, y donde el interés central está ubicado en el grupo de pares, el barrio y la calle. Si bien en muchos de los empleos a los que acceden (como albañiles o peones de carga y descarga de materiales, por ejemplo) la fuerza física y la masculinidad son elementos valorizados, al mismo tiempo, las condiciones de empleo, caracterizadas por la precariedad e inestabilidad, impiden que "lo varonil" pueda efectivamente realizarse en el ejercicio de esa actividad. Por el contrario, el estar con el grupo de pares, en la calle, en el barrio, resulta alternativamente el modo simbólico desde el cual afirmar la propia masculinidad, principalmente en relación al ejercicio del poder 
y a la adquisición de la aceptación de su entorno. El ideal masculino hegemónico, por lo tanto, es aceptado, pero también vivenciado de un modo frustrante por la incapacidad de cumplirlo completamente, al mismo tiempo en que se cuestiona el desgaste físico que conlleva.

El último grupo de varones comparte un discurso cuestionador de la división sexual del trabajo y argumenta sus desacuerdos respecto del papel tradicional otorgado a los varones. Para ellos, la esfera central de la vida es el estudio, y la inserción laboral es un proceso subsidiario de aquel otro proyecto central. Como se vio, la continuidad de estudios superiores es un recorrido más presente entre estos jóvenes. Los varones de este grupo comparten una percepción de sí mismos como distintos a los varones de su entorno, a los cuales, para ganar su aceptación, deben mostrarles pautas de conducta y códigos de lenguaje típicos del género.

A partir de aquí, se puede retornar a las inquietudes que se planteaban al comienzo del artículo, ahora formulando nuevas preguntas: ¿de qué manera interviene un Centro de FP cuando, a través de sus cursos, refuerza el ideal masculino de la provisión, pero en contextos en donde el empleo formal no es una oportunidad real entre los jóvenes? ¿De qué forma se problematizan, en términos del padecimiento físico que conllevan, los empleos típicamente masculinos? ¿De qué modo se aborda la relación y compatibilización entre estudio y trabajo en los Centros de FP? ¿Qué ampliación de horizontes generan estas propuestas educativas en sectores con escasas experiencias en el empleo formal y bajos niveles educativos?

De algún modo, el análisis realizado lleva a plantear que urge desnaturalizar, en el nivel de la FP, la reproducción de las desigualdades de género, pero no únicamente con el público femenino sino también con el masculino -cuestión que todavía aún hoy no se prioriza entre las agendas de género-. Si bien recientemente se ha avanzado en la incorporación de perspectivas de género en las instancias educativas de formación laboral (Bloj, 2017; Millenaar, 2017b), en general, las estrategias que se implementan apuntan a promocionar, acompañar y fortalecer las trayectorias educativas de las mujeres. En tal sentido, los abordajes de género ETP generalmente omiten estrategias concretas de trabajo con los alumnos varones.

En este artículo, se pretendió contribuir en ese camino, mostrando la diversidad de experiencias de masculinidad que participan de la FP, y los sentidos heterogéneos que se construyen en relación a la formación. Conocer esta diversidad es un modo de complejizar el rol de estos dispositivos en relación a la socialización ocupacional y de género, y que evidencia el trabajo de intervención amplio y flexible que se requiere, en pos de poder "pensar" a los varones que se tienen en frente y así contribuir a desnaturalizar las desigualdades de género que aún persisten.

\section{REFERENCIAS}

Alabarces, P. y Garriga Zucal, J. (2008). El aguante: una identidad corporal y popular. Intersecciones en antropología, 9, 275-289.

Assusa, G. (2016). Saberes laborales y disposiciones sociales. Capital cultural y estrategias de inserción laboral entre jóvenes varones de clases populares de Gran Córdoba (pp. 123-141). En M. Busso y P. E. Pérez (Coords.), Caminos al trabajo: el mundo laboral de los jóvenes durante la última etap a del gobierno kirchnerista. Buenos Aires: Miño y Dávila.

Bates, I. (1991). Closely observed training: an exploration of links between social structures, training and identity. International studies in sociology of education, 1, 225-243.

Bloj, C. (2017). Trayectorias de mujeres Educación técnico-profesional y trabajo en la Argentina. Santiago de Chile: CEPAL. Recuperado de https://www.cepal.org/es/publicaciones/41230-trayectorias-mujeres-educacion-tecni co-profesional-trabajo-la-argentina

Bourgois, P. (2010). En busca de respeto: vendiendo crack en Harlem. Buenos Aires: Siglo XXI.

Connell, R. y Messerschmidt, J. (2005). Hegemonic masculinity: rethinking the concept. Gender \& society, 19(6), 829 -859 .

Connell, R. (1995). Masculinities. Berkeley: University of California Press. 
Jiménez Guzmán, L. y Tena Guerrero, O. (2007) (Coords.). Reflexiones sobre masculinidades y empleo. Cuernavaca: UNAM.

Lamamra, N. (2011). La formationprofessionnelle en alternance, un lieu de transmission des normes de genre? Psychologie du travail et des organisations, 17 (4), 330-364.

Medan, M. (2009). Aportes conceptuales para indagar sobre las masculinidades en un programa de prevención del delito. En XXVII Congreso de la Asociación Latinoamericana de Sociología y VIII Jornadas de Sociología de la Universidad de Buenos Aires, Buenos Aires, Argentina. Recuperado de http://cdsa.aacademica.org/000-062/18 54.pdf

Medan, M. (2013). Los sentidos del riesgo en los programas sociales (pp. 99-127). En V. LLovet (Coord.), Sentidos de la exclusión social. Beneficiarios, necesidades y prácticas en politicas sociales para la inclusión de niños y jóvenes. Buenos Aires: Biblos.

Millenaar, V. (2014). ¿Capacitar para la competitividad o promover los derechos? Retóricas de la formación profesional desde un análisis de género. Propuesta educativa, 23(41), 99-108, Recuperado de http://www.propuestaeducat iva.flacso.org.ar/archivos/jovenes/23.pdf

Millenaar, V. (2017a). Trayectorias educativo-laborales de varones y mujeres jóvenes de sectores populares que participan de dispositivos de formación para el trabajo. (Area Metropolitana de Buenos Aires, 2008-2013). Entre la profesionalización, la acumulación y la socialización. Tesis de Doctorado en Ciencias Sociales, Universidad de Buenos Aires, Buenos Aires.

Millenaar, V. (2017b). Políticas de empleo con enfoque de género: formación laboral en oficios no tradicionales para mujeres. Cadernos Pagu [online]. 51, e175114. Recuperado de http:// dx.doi.org/10.1590/18094449201700510014

Millenaar, V. y Jacinto, C. (2015). Desigualdad social y género en las trayectorias laborales de jóvenes de sectores populares. El lugar de los dispositivos de inserción (pp.73-100). En L. Mayer, D. Llanos y R. Unda Lara (Comps.), Socialización escolar. Procesos, experiencias y trayectos. Ecuador: Abya Ayala- Universidad Politécnica Salesiana CINDE y CLACSO, Recuperado de http://www.unsam.edu.ar/observatorio-jovenes/articulos/articulo4.pdf

Miranda, A. y Corica, A. M. (2015). Las actividades laborales y extra-escolares de jóvenes de la escuela secundaria en la Argentina de principios del Siglo XXI. Perfiles educativos. 37 (148), 100-118. Recuperado de http://www.sci elo.org.mx/scielo.php?pid=S0185-26982015000200007\&script=sci_abstract

Pérez, P. E. (2016). Jóvenes, trabajo y desigualdades de género en Argentina (2003-2014) (pp. 33-50). En M. Busso y P. E. Pérez (Coords.), Caminos al trabajo: el mundo laboral de los jóvenes durante la última etapa del gobierno kirchnerista. Buenos Aires: Miño y Dávila.

Scott, J. (2008). Unanswered questions. American historical review, 113 (5), 1422-1430, Recuperado de http://www .culturahistorica.es/scott/unanswered.pdf

Segato, R. (2003). Las estructuras elementales de la violencia. Ensayos sobre género entre la antropología, el psicoanálisis $y$ los derechos humanos. Buenos Aires: Prometeo UNQui.

Silba, M. (2011). “Te tomás un trago de más y te creés rambo": políticas, representaciones y sentido común sobre varones jóvenes (pp. 229-267). En S. Elizalde (Coord.),Jóvenes en cuestión. Configuraciones de género y sexualidad en la cultura. Buenos Aires: Editorial Biblos.

Skeggs, B. (1997). Formations of class and gender. London: Sage.

Subirats, M. (1986). Niños y niñas en la escuela: una exploración de los códigos de género actuales (pp. 381-392). En M. Fernández Enguita (Ed.), Marxismo y sociología de la educación Madrid: Akal,

Willis, P. (1998 [1977]). Aprendiendo a trabajar: como los chicos de clase obrera consiguen trabajos de clase obrera. Madrid: Akal. 


\section{Notas}

1 En la investigación mencionada se muestra que los varones egresados de FP suelen tener mejores inserciones luego de los cursos que sus pares mujeres, y en mayor medida, una movilidad hacia la formalidad, inserción en establecimientos de mayor tamaño, ocupaciones de mayor calificación y reducción de la sub-ocupación horaria (Millenaar y Jacinto, 2015).

2 La FP en Argentina es un nivel educativo orientado al trabajo y a la formación en oficios, organizado en cursos o módulos, de duración variada, dependiente de cada una de las jurisdicciones del país. Se encuentra destinado a jóvenes y adultos de más de 18 años con nivel de primario completo o más. Los tres Centros de FP seleccionados para la investigación (situados en lo que se conoce como AMBA, Área Metropolitana de Buenos Aires) son contrastantes entre sí, tanto en relación a sus modos de gestión, como en relación a sus abordajes de género (para mayores referencias ver: Millenaar, 2017a). Cabe señalar que, en la provincia de Buenos Aires, al momento de la realización del trabajo de campo, el área de FP dependía de la Dirección Provincial de Educación Técnico-Profesional. Por su parte, en la Ciudad de Buenos Aires la FP depende de la Gerencia Operativa de Educación y Trabajo. El Instituto Nacional de Educación Tecnológica (INET) es el organismo, a nivel nacional, que marca los lineamientos del nivel.

3 Como se ha mencionado, los jóvenes varones que se analizan en este artículo conforman la muestra de jóvenes tomadas en la tesis doctoral (Millenaar, 2017a), que ha seguido criterios teóricos en su conformación, considerando relevante tomar un rango etario amplio para reconstruir trayectorias laborales de diferentes duraciones. Todos los varones analizados en este artículo egresaron de algún curso de FP de los centros seleccionados (uno situado en Troncos del Talar, Partido de Tigre, otro en Caseros, Partido de 3 de febrero y otro en el barrio de Barracas, Ciudad de Buenos Aires). En su mayoría, provienen de hogares en donde ninguno de sus padres alcanzó mayor nivel educativo que secundario incompleto. De este modo, se trata de un grupo de varones proveniente de hogares con bajo clima educativo, que constituye un proxi de hogares de bajo nivel socioeconómico (para mayores referencias ver: Millenaar, 2017a).

4 "Bancarsela", en la jerga juvenil en Argentina, refiere a la capacidad de soportar determinadas condiciones o situaciones por lo general adversas o penosas.

5 El primer grupo está conformado por 11 varones, todos de entre 18 y 25 años, la mitad de ellos con un nivel educativo de secundario incompleto o menos, un tercio de ellos residiendo con su propia familia (dos de ellos ya son padres) que en su mayoría- muestran una trayectoria laboral continua, y que, al momento de la entrevista, se encontraban casi todos ocupados en un empleo formal.

6 El segundo grupo está conformado por 10 varones, de edades diversas que van de los 18 a los 32 años, en su mayoría con nivel educativo de secundario incompleto o menos y residiendo con sus familias de origen (sólo uno es padre). Todos muestran trayectorias laborales discontinuas y, al momento de la entrevista la mayoría se encontraba ocupado en el empleo informal (el resto, desocupado).

7 "El trabajo es cosa buena

Es lo mejor de la vida

Pero la vida es perdida

Trabajando en campo ajeno.

Unos trabajan de trueno

Y es para otro la llovida”

(Atahualpa Yupanqui, Coplas del Payador Perseguido)

8 "Ponerse pillo" en la jerga juvenil significa ganar astucia, ser inteligente o sagaz.

9 "Tener bondi" significa en la jerga juvenil estar en problemas, estar peleado con tal o cual. "Fierros" refiere a la portación de armas.

10 "Ponerse las pilas" es una expresión que refiere a movilizar la propia voluntad, comprometerse. Por su parte,"rescatarse", refiere a la responsabilización frente al propio modo de conducirse.

11 Darse "maña" significa saber desenvolverse, resolver un problema.

12 "Trampa" se refiere a cometer una infidelidad.

13 El tercer grupo está conformado por 9 varones, de edades concentradas entre los 18 y 23 años, en su mayoría con secundario completo y más, residentes casi en su totalidad con sus familias de origen (sólo uno es padre). Se destaca en este grupo el recorrido educativo que se realiza sin interrupciones (no repiten de año o dejan los estudios), así como la ausencia, en su mayoría, de experiencia laboral antes de asistir a la formación. La mayoría, al momento de la entrevista se encontraban ocupados en empleos formales. 